Psychotherapeut 2021 - 66:314-323 https://doi.org/10.1007/s00278-021-00490-w Angenommen: 4. Januar 2021

Online publiziert: 16. Februar 2021

(c) Der/die Autor(en) 2021

Matthias Michal' · Kristin Osborn ${ }^{2}$

${ }^{1}$ Klinik und Poliklinik für Psychosomatische Medizin und Psychotherapie, Universitätsmedizin Mainz, Mainz, Deutschland

${ }^{2}$ Department of Psychiatry, Harvard Medical School, Boston, USA

\title{
Die Affektphobietherapie
}

\section{Psychodynamisches transdiagnostisches manualisiertes Behandlungsmodell}

Behandlungen effektiver zu machen, vorwiegend durch eine Fokussierung der Interventionen (Balint et al. 2013). Die Arbeitsgruppe hatten einen engen Bezug zur Objektbeziehungs- und Bindungstheorie und betonte die Bedeutung des emotionalen Erlebens. Mitte der 1960erJahre wurde die Arbeitsgruppe von David H. Malan (1922-2020) weitergeführt. Malan selbst befand sich noch bei Michael Balint (1896-1970) und Donald Winnicott (1896-1971) in der Lehranalyse (Eppel 2018). Auf Malan (1963, 1979 orig., zit. nach Malan 2013) geht die Ausarbeitung eines zentralen Instruments der STDP zurück, das auch in der APT die zentrale Rolle für das Verständnis der Symptome und die Strukturierung der Interventionen spielt: das Konfliktund das Personendreieck (• Abb. 1). Nach Malan repräsentieren diese beiden Dreiecke das „universelle Prinzip der psychodynamischen Psychotherapie“. Konflikthafte Gefühle ( $F$, „feeling“) triggern Angst ( $A$, ,anxiety/inhibition“) und werden deshalb abgewehrt $(D$, „defense“). Diese maladaptiven Reaktionsmuster wurden in Abhängigkeitsbeziehungen aus der Vergangenheit $(P$, „past person“) erlernt und werden in gegenwärtigen Beziehungen $(C$, „current person") wiederholt, aufrechterhalten und auf den Therapeuten ( $T$, ,therapist/ transference") übertragen.

Die APT wurde von Leigh McCullough (1945-2012) entwickelt. McCullough entschied sich zu Beginn ihrer Karriere zunächst für eine verhaltenstherapeutische Ausbildung bei Joseph Cautela, einem fortschrittlichen Verhaltenstherapeuten, der im Konzept der ver- deckten Konditionierung („,covert conditioning“, Cautela 1973) die Prinzipien der Lerntheorie mit dem Vorstellungserleben in Verbindung brachte (Cautela 1966). Cautela nutzte erstmals die imaginative Konfrontation, um maladaptives Verhalten zu verändern. Cautela war es auch, der McCullough mit der wegweisenden Monografie Psychoanalysis and Behavior Therapy: Toward an Integration (Wachtel 1977) bekannt machte. In ihrer weiteren beruflichen Entwicklung - so McCullough - erkannte sie die Grenzen ihrer therapeutischen Techniken bei der Behandlung von Patienten mit Persönlichkeitsstörungen und suchte nach Möglichkeiten, ihre psychotherapeutischen Fähigkeiten weiterzuentwickeln. In diesem Kontext besuchte sie als Verhaltenstherapeutin einen Workshop an der Tavistock-Klinik bei Malan, der mithilfe von Videoaufnahmen von Therapiesitzungen die Methode der STDP vermittelte. Malan empfahl ihr - auch der räumlichen Nähe wegen - Habib Davanloo, den Begründer der Intensiven Psychodynamischen Kurzzeittherapie (,intensive short-term dynamic psychotherapy“, ISTDP; Davanloo 2001), als Trainer und Supervisor. Von 1983 bis 1985 nahm sie an seinem videobasierten „core training“ in Manhattan teil. Nachhaltig beeindruckt hatte sie in diesen Workshops, wie durch die konsequente und beharrliche Bearbeitung der Abwehr - also durch Reaktionsverhinderung im verhaltenstherapeutischen Sinne - auch komplexe und bisher therapieresistente Störungen erfolgreich behandelt werden konnten (McCullough et al. 2019). Dies war jedoch nicht die einzige in- 
Das Konfliktdreieck

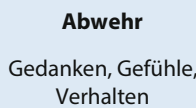

D

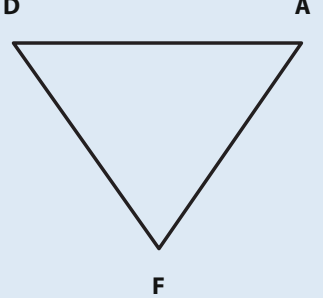

Adaptive Gefühle/Aktivierung

Trauer, Ärger, Nähe, positive

Selbstgefühle, Interesse,

Begeisterung, Genuss, Freude, sexuelle Lust

Angst

A
Das Personendreieck

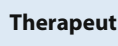

T

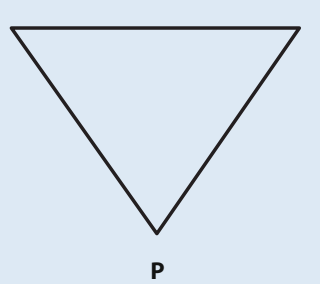

Personen der Vergangenheit

Eltern, Geschwister, Verwandte, Lehrer, Kindheitsfreunde

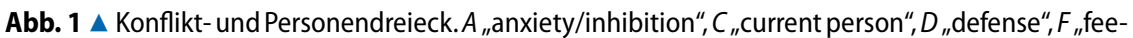
ling", $P$ "past person", $T$ "therapist/transference"

tensive Begegnung mit der Tiefenpsychologie. Zuvor war sie von 1980 bis 1983 im Rahmen eines Forschungsprojekts an der Harvard Universität von George Vaillant (2011) mit der Operationalisierung und Auswertung von Abwehrmechanismen betraut worden. So vorbereitet entwickelte sie den zentralen Gedanken des APT-Modells, dass psychische Störungen als Folge von Affektphobien verstanden werden können: So wie eine Person sich davor fürchtet, eine Brücke zu betreten, so kann eine Person Angst davor haben, bestimmte Affekte zu erleben und diese dann phobisch vermeiden, was mit entsprechend schädlichen Konsequenzen einhergeht. Das Vermeidungsverhalten entspricht der Abwehr im psychodynamischen Sinne. Wenn beispielsweise im Laufe der Entwicklung der Affekt „Wut“ die primäre Bindung gefährdete, löst später das Aufkommen von Wut automatisch Angst und das damit verbundene Abwehrverhalten aus: Beispielsweise wird die Emotion „Wut“ dann gegen das eigene Selbst in Form von Selbstkritik gewendet, anstatt der Abgrenzung und Selbstbehauptung zu dienen. Dies kann zu einer Depression führen: „Anstatt den anderen niederzuschlagen, schlägt man sich selbst nieder." Im APT-Modell wird durch die Desensibilisierung des Affekts der „Wut“ diese Emotion wieder für die Person auf adaptive Weise nutzbar, anstatt auf maladaptive Weise vermieden bzw. abgewehrt zu werden. Das Konzept der Affektphobie stellt damit eine Umformulierung der psychodynamischen Konflikttheorie mithilfe der Konzepte der Lerntheorie dar. Hierdurch können bewehrte Techniken und Konzepte der verhaltenstherapeutischen Behandlung von Phobien wie das der systematischen Desensibilisierung in einem psychodynamischen Behandlungskontext anwendet werden (McCullough et al. 2003, 2019; McCullough Vaillant 1997).

\section{Behandlungsprinzipien}

Die zentralen Behandlungsprinzipien der APT sind (McCullough et al. 2019), dem Patienten zu helfen,

1. das gesamte Spektrum seiner Gefühle erleben und adaptiv ausdrücken zu können;

2. Mitgefühl mit sich und anderen $\mathrm{zu}$ haben;

3. $\mathrm{zu}$ einer gesunden Balance von Autonomie und Abhängigkeit zu finden und

4. die Behandlung so effizient wie möglich zu gestalten.
Die therapeutische Haltung ist gekennzeichnet durch eine partnerschaftliche Beziehung auf „Augenhöhe" und die Betonung des patientenseitigen Willens zur Zusammenarbeit sowie die aktive Bearbeitung der Abwehr im Gegensatz zur freien Assoziation, um maligne Regressionen und die Entfaltung einer Übertragungsneurose zu verhindern. Verhaltenstherapeuten werden vieles an dieser therapeutischen Haltung auch in dem „cognitive behavioral analysis system of psychotherapy" wiederfinden (McCullough 1984).

Die APT zählt zur Familie der STDP. „Short term“ ist hier jedoch nicht gleichzusetzen mit dem Terminus „Kurzzeit“ der Psychotherapierichtlinie. Die typische Sitzungsdauer beträgt $50 \mathrm{~min}$ mit einer Frequenz von einer bis 2 Sitzungen/ Woche. Die APT-Behandlungen dauern so lange wie notwendig. In Abhängigkeit von der Komplexität der Störung können Behandlungen sich über 100 und mehr Stunden erstrecken. Die APT ist für das gesamte Spektrum psychischer Störungen geeignet; es gibt keine störungsspezifischen Kontraindikationen (McCullough et al. 2003, 2019). Das Manual ist prozessorientiert. Die Interventionen antworten auf die klinischen Phänomene, die mithilfe des Konfliktund des Personendreiecks strukturiert werden (•Abb. 1).

\section{Bedeutung der Affekte}

Nachfolgend werden die Begriffe Affekt, Emotion und Gefühl weitgehend synonym verwendet. In der APT stehen die Affekte im Mittelpunkt der Therapie, weil psychische Störungen als Folge von Affektphobien betrachtet werden (McCullough et al. 2019). Affekte gelten als das primäre Motivationssystem des Menschen (Demos 2019; Grecucci et al. 2017; Tomkins et al. 1995). Affekte geben Orientierung. Sie stellen eine Art innerer Kompass dar. Sie wahrzunehmen bedeutet, mit der inneren Stimme in Kontakt $\mathrm{zu}$ sein, sich lebendig zu fühlen. Affekte adaptiv ausdrücken zu können, ist die Voraussetzung für gelingende soziale Beziehungen (McCullough et al. 2019; McCullough Vaillant 1997). In der Behandlung werden Patienten fortwährend 
angeleitet, sich der eigenen Gefühle bewusst zu werden. Dies bedeutet erstens, sie im Körper zu spüren, zweitens sie richtig zu benennen und drittens sich der damit verbundenen Handlungsimpulse bewusst zu werden. Auch wenn es in der APT als essenziell betrachtet wird, alle Gefühle fühlen zu können, geht es nicht darum, Gefühlen blind $\mathrm{zu}$ folgen bzw. diese auszuagieren, sondern sich der inneren emotionalen Welt bewusst zu werden, um dadurch adaptive Entscheidungen treffen zu können. Denn man kann nur regulieren, was man bewusst wahrnimmt.

Da für den Ausdruck der Affekte die Differenzierung adaptiver von maladaptiven Affekten wichtig ist, haben McCullough et al. (2003, 2019) entsprechende Kriterien herausgearbeitet. Wichtige Unterscheidungskriterien sind die Konsequenzen des Affektausdrucks und das körperliche Erleben des Affekts. Ein adaptiver Affekt kann im Körper wahrgenommen werden; sein Ausdruck ist befreiend und stellt eine gesunde Reaktion dar. Maladaptive Affekte stehen in den Diensten der Abwehr; der Ausdruck ist interpersonell destruktiv und mehrt Missverständnisse, Einsamkeit und Hoffnungslosigkeit. Beispielsweise können Tränen oder das Wort Trauer eine maladaptive Abwehr darstellen. Die Unterscheidung lässt sich durch die Exploration des körperlichen Erlebens und der damit verbundenen Vorstellungen aufklären. Echte Tränen und echte Trauer sind, wenn auch schmerzlich, so doch letztendlich befreiend und stärken die Verbundenheit. Maladaptive Tränen verdecken oft Wut und stehen für eine Position der selbstquälerischen Hilfsund Hoffnungslosigkeit oder spiegeln massive Selbstkritik wider. Diese Tränen vergrößern die Qual, wirken nicht befreiend und stellen auch kein progressives therapeutisches Ereignis dar, sondern stehen in den Diensten der Abwehr und halten die Störung des Patienten aufrecht.

\section{Konfliktdreieck und Personen- dreieck}

Das Konfliktdreieck beschreibt die Psychodynamik der Symptome, das Perso-

Psychotherapeut 2021 - 66:314-323 https://doi.org/10.1007/s00278-021-00490-w

(c) Der/die Autor(en) 2021

\section{Michal $\cdot$ K. Osborn}

\section{Die Affektphobietherapie. Psychodynamisches transdiagnostisches manualisiertes Behandlungsmodell}

\section{Zusammenfassung}

Hintergrund. Ein zentraler Faktor psychischer Gesundheit ist die Fähigkeit, Affekte wahrnehmen und adaptiv regulieren zu können. Die Verbesserung dieser Fähigkeit ist ein Ziel der psychodynamischen Psychotherapie. Psychodynamische Behandlungsmodelle, die sich aus der gut validierten Tradition der "short-term dynamic psychotherapy" (STDP) ableiten und die heute unter dem Dach der International Experiential Dynamic Therapy Association (https://iedta.net/) versammelt sind, legen auf besondere Weise den Behandlungsfokus auf das emotionale Erleben. Ein derartiges psychodynamisches transdiagnostisches Behandlungsmodell ist die Affektphobietherapie (APT).

Ziel der Arbeit (Fragestellung). Ziel der Übersicht ist es, den Leser mit dem Behandlungsrational und der Evidenz der APT vertraut zu machen.
Material und Methoden. Es werden die theoretischen Grundlagen der APT, typische Interventionen und Prozessinstrumente beschrieben. Die Evidenz des APT-Modells wird als narrative Übersicht der Studienlage dargestellt.

Ergebnisse und Schlussfolgerung. Die APT ist ein evidenzbasiertes psychodynamisches Behandlungsmodell. Das Rational ist schulenübergreifend. Ein Selbsthilfeprogramm auf der Basis der APT wird von Patienten gut aufgenommen. Manualisierung, Fertigkeitenorientierung und Prozessinstrumente machen es zu einem vielversprechenden Modell für die Aus- und Fortbildung.

\section{Schlüsselwörter}

Emotionen · Desensibilisierung · Konflikt, psychodynamisch · Zwischenmenschliche Beziehungen $\cdot$ Manualisierung

\section{Affect phobia therapy. Psychodynamic transdiagnostic manualized treatment model}

\section{Abstract}

Background. A central factor of mental health is the ability to experience affects and to regulate them adaptively. The improvement of this capacity is a treatment aim of psychodynamic psychotherapy. Psychodynamic treatment models derived from the wellvalidated tradition of short-term dynamic psychotherapy (STDP), which are gathered under the umbrella of the International Experiential Dynamic Therapy Association (https://iedta.net/), have a special focus on emotional experiencing in their treatment. One such psychodynamic transdiagnostic treatment model is affect phobia therapy (APT).

Objective. The aim of the review is to introduce the reader to the treatment rationale and evidence for APT.
Material and methods. The theoretical principles of the APT treatment model, typical interventions and process instruments are described. The evidence for the APT model is presented as a narrative review.

Results and conclusion. The APT is an evidence-based psychodynamic treatment model. The treatment approach is integrative. A self-help program based on APT is wellreceived by patients. Manualization, skill orientation and process instruments make it a promising tool for education and training.

\section{Keywords}

Emotions - Desensitization · Conflict, psychodynamic · Interpersonal relations . Manualization nendreieck die Entstehung und Aufrechterhaltung der maladaptiven Reaktionsmuster in den primären und aktuellen Beziehungen (• Abb. 1).

Symptome werden im Konfliktdreieck als Folge der Abwehr adaptiver Affekte (beispielsweise Wut, Liebe, Trauer, positive Selbstgefühle, Sexualität) verstanden, die in der individuellen Entwicklungsgeschichte konflikthaft wurden und deshalb Angst und Abwehr auslösen (Vaillant 1997). Das Konfliktdreieck stellt damit eine Ableitung von Freuds zweiter Angsttheorie dar, wobei die Funktion der "Triebregungen“ durch die der Affekte ersetzt wurde (Freud 1991; Vaillant 


\section{Fallbeispiel 1 Anwendung des Konfliktdreiecks}

Patient: Ich habe meinem Kollegen gesagt, dass es mir nicht recht ist, dass er schon wieder einen Brückentag nimmt und ich schon wieder leer ausgehe (Ärger/ Selbstbehauptung $\hat{=}$ adaptives Gefühl), aber dann fühlte ich mich plötzlich unsicher (Angst [A-Pol im Konfliktdreieck]), es war mir peinlich (Scham-Angst und Abwehr in Form von Selbstverurteilung und Projektion der Verurteilung), und ich machte einen Rückzieher (Abwehr: Vermeidung, Unterwerfung).

Erläuterung: In dieser kurzen Vignette stellt Ärger das abgewehrte adaptive Gefühl dar (F-Pol im Konfliktdreieck), das den Patienten bewegte, sich kurz gegenüber dem Kollegen zu behaupten. Auf die Mobilisierung des Ärgers folgte jedoch Angst (A-Pol im Konfliktdreieck) und setzte dann die Abwehr (D-Pol im Konfliktdreieck) in Form von Selbstkritik, Projektion der Kritik auf andere und Unterwerfung in Gang. Die Abwehr bedingt das maladaptive symptomatische Verhalten.

1997). Die Anwendung des Konfliktdreiecks wird in Fallbeispiel 1 aufgezeigt.

Affektphobien entwickeln sich in der Beziehung zu den primären Bezugspersonen, denn Gefühle sind immer mit Beziehungen und Beziehungserfahrungen assoziiert (Bowlby 2010; Vaillant 1997). Dies wird im Personendreieck verdeutlicht. Affektphobien werden in den primären Beziehungserfahrungen erlernt bzw. konditioniert ( $\mathrm{P}$, „past persons" [Personen der Vergangenheit]) und in den heutigen Beziehungen ausgelebt (C, „current persons“ [aktuelle Beziehungen] und $\mathrm{T}$, „transference“ [Übertragung]).

In diesem Modell kommen Menschen als Säuglinge unbelastet von neurotischen Konflikten auf die Welt und sind kompetent darin, über den Ausdruck ihrer Gefühle das Gegenüber wissen zu lassen, was sie wollen. Sie schreien, wenn etwas nicht passt, und hören nicht damit auf, bis ihre Eltern herausgefunden haben, was sie brauchen. Unglücklicherweise lernen Kinder aber auch, dass der Ausdruck bestimmter Gefühle mit der Gefahr verbunden ist, die „Liebe“ der primären Bezugsperson zu verlieren, was Angst auslöst. Über den Prozess der Konditionierung ruft der
Fallbeispiel 2 für die Umstrukturierung der Abwehr

$P$ : (Patient wendet den Blick ab, schaut längere Zeit auf den Boden, während er spricht.)

$T$ : Fällt Ihnen auf, wie Sie den Blick abwenden, während Sie mit mir sprechen [Markieren der Abwehr gegen emotionale Nähe]? Was würde passieren, wenn Sie mich anschauen? Wollen Sie es einmal versuchen? [Herausforderung der Abwehr].

$T$ : Was sind die Hauptprobleme, für die Sie meine Hilfe suchen?

$P$ : Ich bin fertig, ich habe keine Energie mehr, und nichts macht mir mehr Freude, seit meine Frau mich vor 6 Wochen verlassen hat. Sie ist einfach ausgezogen und wohnt bei einem anderen Mann.

$T$ : Welche Gefühle hat es bei Ihnen ausgelöst, dass Ihre Frau Sie verlassen hat?

P: Ich weiß nicht [Abwehr: Hilflosigkeit oder Vermeidung von emotionaler Nähe] ---(kurzes Schweigen), ich denke, ich bin so ein Idiot [Abwehr: Wendung gegen das Selbst]. $T$ : Wenn Sie sich hier als Idiot bezeichnen, hat dies etwas Verletzendes, so als ob Sie sich selbst verletzen [Spiegelung der Abwehr]. Wenn Sie sich nicht selbst angreifen, welche Gefühle würden dann gegenüber Ihrer Frau in Reaktion auf das Verlassenwerden auftreten [Herausforderung der Abwehr]?

Affekt später automatisch Angst und das entsprechende Abwehrverhalten hervor (McCullough Vaillant 1997; Vaillant 1997). In einer gesunden Entwicklung kommt es zu keiner massiven Angst vor den eigenen Gefühlen, sondern das Kind lernt, Emotionen auf der Basis einer sicheren Bindung zu regulieren (Fonagy et al. 2010; McCullough et al. 2019).

\section{Interventionsstrategien}

In der APT werden 3 Kategorien von Interventionen unterschieden, die abhängig vom therapeutischen Prozess und dem Funktionsniveau des Patienten angewendet werden. Orientierung über den Prozess gibt das Konfliktdreieck. Der Therapeut prüft, ob gerade Abwehr, Angst oder der adaptive Affekt/ Impuls im Vordergrund steht und passt daraufhin seine Interventionen an.
Fallbeispiel 3 für die Umstrukturierung der Affekte

$T$ : Sie sagen, das hat Sie wütend gemacht. Wie fühlen Sie die Wut in Ihrem Körper? [ $T$ fokussiert auf das körperliche Erleben der Emotion].

$P$ : (sich im Stuhl aufrichtend und die Fäuste ballend) Da ist ein Hitzegefühl im Bauch, das hochkocht [Psychomotorik und Introspektion des Patienten zeigen, dass er die Wut körperlich erleben kann, ohne dabei ein Übermaß an Angst zu erleben].

$T$ : Mit welchem Impuls ist die Wut verbunden? Wie würden Sie, wenn es nur noch diese Wut gäbe und nichts, was sie zurückhält, wie würden Sie in der Fantasie die ganze Wut mit voller Wucht auf die Frau kommen lassen? [Der Therapeut legt nun den Fokus auf den mit dem Affekt der Wut verbundenen Handlungsimpuls. Dies ist möglich, weil der Patient den Affekt der Wut innerlich auf der Basis einer sicheren Beziehung zum Therapeuten erleben kann.].

$P$ : (mit fester Stimme, Fäuste ballend und mit angedeuteten Faustschlägen) Ich würde ihr immer und immer wieder in die Fresse schlagen, bis das Gesicht ein blutiger Brei ist, bis sie tot ist [Erleben der Handlungsimpulse unbewusster mörderischer Wut ohne

Angstsymptome].

$T$ : Wenn Sie dann der Leiche in die Augen schauen, welche Farbe haben die Augen, wen sehen Sie da?

$P$ : Meine Mutter (--- fängt an zu schluchzen).

\section{Umstrukturierung der Abwehr}

Hierbei zielen die Interventionen darauf $\mathrm{ab}$, dem Patienten zu helfen, sich seiner Abwehr bewusst zu werden, d. h., die Abwehr zu erkennen, einschließlich deren destruktiver Konsequenzen, sie aufzugeben und durch gesündere Reaktionen zu ersetzen (Fallbeispiel 2). In der Sprache der Verhaltenstherapie wären dies Interventionen zur Reaktionsverhinderung.

\section{Umstrukturierung der Affekte}

Diese Interventionen zielen auf die Reduktion der Angst vor und dem Erleben der bisher konflikthaften Affekte. Dabei geht es im ersten Schritt um das volle Erleben der Affekte und im zweiten Schritt um deren angemessenen Ausdruck. Das volle Erleben der Affekte entspricht in der Sprache der Verhaltenstherapie der Exposition mit dem phobischen Stimulus. Die graduierte Exposition der angstmachenden Affekte in einer sicheren Beziehung bei gleichzeitiger Blockierung 


\section{Fallbeispiel 4 Interventionen für die Umstrukturierung der Selbstvorstellung}

$T$ : Können Sie sich vorstellen, Sie hören die Geschichte, die Sie mir gerade erzählt haben, im Radio. Welche Gefühle würde diese Geschichte bei Ihnen gegenüber der Person, die all das durchgemacht hat, hervorrufen [Perspektivenwechsel]?

$P: \mathrm{Hm}$... ich wäre traurig und würde sie trösten.

$T$ : Können Sie jetzt und hier Mitgefühl sich selbst gegenüber aufbringen? [Exposition mit positiven Gefühlen dem Selbst gegenüber].

$P$ : Nein, ich komme mir dumm vor, ich würde mich für arrogant halten. [Abwehr].

$T$ : Also, wenn es darum geht, sich selbst zu akzeptieren und zu lieben, dann kommen Sie sich also dumm und arrogant vor. Sind Sie sich bewusst, dass Sie sich selbst abwerten, wenn es darum geht, sich zu akzeptieren und wertzuschätzen? [Bewusstmachen der Abwehr].

$P$ : Nein.

$T$ : Was passiert gerade in Ihrem Körper [Intervention zur Angstregulation], während wir darüber sprechen, wie Sie sich selbst abwerten, anstatt sich mit Mitgefühl und Wertschätzung zu begegnen?

$P$ : Ich fühle mich angespannt [ $\hat{=}$ Angst].

$T$ : Ja, es macht lhnen Angst, und etwas verkrampft sich in Ihnen $[A$, Angstpol], Sie werten sich selbst als dumm und arrogant ab [D, Abwehr], anstatt sich zu akzeptieren und sich mit Mitgefühl und Wertschätzung zu begegnen [F, adaptive Emotion]. Das klingt schmerzhaft. [Formulierung des zentralen Konflikts mit dem Konfliktdreieck].

$P$ : Das ist es auch.

$T$ : Wollen Sie es ändern? Wollen Sie lernen, sich selbst gegenüber mitfühlend und liebevoll zu sein? [ $T$ motiviert den Patienten, sich gegen die Abwehr zustellen].

$P$ : Ja, deshalb bin ich hier. Ich bin zu hart zu mir selbst und erwarte zu viel. Ich bin erschöpft und ausgebrannt.

$T$ : Beginnen wir damit herauszufinden, was das absolut Schlimmste ist, was Ihnen passieren kann, wenn Sie sich selbst gegenüber Mitgefühl und Wertschätzung aufbringen. [Exploration der Angst und Abwehr].

der Abwehr bzw. des Vermeidungsverhaltens und der Regulation der dabei aufkommenden Angst führt zur Desensibilisierung des phobischen Affekts. Dieser Affekt macht dem Patienten nun keine Angst mehr und muss deshalb nicht mehr abgewehrt werden, sondern steht ihm wieder zur Verfügung (McCullough und Osborn 2004). Für die erfolgreiche
Fallbeispiel 5 für die Umstrukturierung der Objektvorstellung

$P$ : Sie kommen mir so kalt und distanziert vor, wie mein Vater (mit gereizter Stimme)! [Übertragung, gleichzeitig projektiver Ärger]. $T$ : Das hört sich sehr unangenehm an. Können Sie mir erzählen, wie es kommt, dass Sie sich mich so wahrnehmen? [Untersuchung der Objektwahrnehmung und Realitätsprüfung]. $P$ : Sie fragen nur und erzählen nichts von sich. $T:$ Ja, das stimmt. Aber ich frage mich, ob Sie sich noch andere Gründe für mein Verhalten vorstellen können, als dass ich darauf aus bin, Ihnen gegenüber kalt und distanziert zu sein? [Intervention zur Verbesserung der Realitätsprüfung].

$P$ : (nickt) Ich weiß Sie sind Therapeut, es geht hier um mich.

$T$ : Ist es für Sie in Ordnung, wenn wir nun Ihre Gefühle untersuchen, die bei Ihnen ausgelöst werden, wenn ich „kalt und distanziert wie ihr Vater" erscheine? [Nachdem der Patient den Therapeuten von der Vaterübertragung kognitiv unterscheiden kann, wird es möglich, die dadurch ausgelösten Gefühle zu untersuchen. Erst die Verarbeitung dieser Gefühle wird es dem Patienten ermöglichen, eine realistischere Objektwahrnehmung aufzubauen.]

Desensibilisierung ist es notwendig, dass der Patient nicht nur über die Gefühle spricht, sondern sie voll erlebt (d.h., den Affekt korrekt benennt, ihn im Körper spürt und den damit verbundenen Handlungsimpuls beschreibt). Voraussetzung für diese Art der Interventionen sind adaptive Selbst- und Objektvorstellungen sowie, eng damit verbunden, eine ausreichende Fähigkeit zur Impulskontrolle. Es geht bei der Umstrukturierung der Affekte darum, in einem sicheren Raum jedes Gefühl innerlich zu erleben und zu untersuchen, wobei je nach Affekttoleranz die Intensität des Gefühls allmählich gesteigert wird. Sehr intensive Aggressionen, auch wenn sie von Personen aus der Gegenwart getriggert wurden, rühren aus frühkindlichen emotionalen Verletzungen. Das Erleben dieser Emotionen mit der vollen Intensität bringt dann meist Erinnerungen an diese Zeit zutage. Im Fallbeispiel 3 könnte sich dann beispielsweise das Gesicht der Freundin in das eines Elternteils verwandeln.

\section{Umstrukturierung der Selbst- und Objektvorstellungen}

Diese Interventionen sind strukturbezogen und zielen darauf ab, Selbstfürsorge und Mitgefühl mit sich selbst aufzubauen und die Beziehungsfähigkeit zu verbessern. Sie umfassen immer auch die Bearbeitung primitiver realitätsverzerrender Abwehrmechanismen wie Projektion, Wendung gegen das Selbst und Spaltung sowie Interventionen zur Umstrukturierung der Affekte, wie das Erleben liebevoller Gefühle (Stolz, Interesse, Nähe, Fürsorge) für sich und andere. Typische Interventionen zur Umstrukturierung der Selbstvorstellungen zielen auf die Anerkennung des eigenen Werts, der eigenen Stärken und Grenzen, der Wahrnehmung und Responsivität gegenüber den Signalen des eigenen Körpers, der Fähigkeit zur Selbstfürsorge und Selbstbehauptung, um die eigenen Bedürfnisse verfolgen zu können (Fallbeispiel 4).

Für die Umstrukturierung der Objektvorstellungen wird untersucht, wie der Patient andere Menschen, einschließlich die Person des Therapeuten, wahrnimmt (Fallbeispiel 5). Dies ist v.a. notwendig bei Problemen mit Intimität, emotionaler Nähe und Beziehungsproblemen. Nur wenig kann in einer Therapie erreicht werden, wenn ein Patient kein Vertrauen und keine Anteilnahme in der therapeutischen Beziehung zulassen kann. Behandlungsziel ist ein reifes, gut integriertes Selbst, das ein gesundes Gleichgewicht von Autonomie und Verbundenheit mit anderen herstellen kann.

\section{Prozessinstrumente}

Für die APT wurden Prozessinstrumente entwickelt, mit der Videosequenzen der Therapiesitzungen ausgewertet werden, und die bei Bedarf in der Prozessforschung und Ausbildung Anwendung finden können: Mithilfe der Rating-Skala „Achievement of Therapeutic Objectives Scale (ATOS) “werden die Fortschritte des Patienten beurteilt (Ryum et al. 2014a, 2014b; Valen et al. 2011): Beurteilt werden auf einer Skala von 1 bis 100 , wie gut der Patient seine maladaptiven Reaktionsmuster (Abwehr) erkennt, das Ausmaß seiner Motivation, dieses maladaptive Verhalten einzustellen, die 
91-100 Hervorragende Identifizierung maladaptiver Muster: Der Therapeut beschreibt in Kooperation mit dem Patienten das maladaptive Reaktionsmuster klar und deutlich, benennt die Abwehrmechanismen und diejenigen Ängste, die zur Abwehr eines bestimmten Gefühls eingesetzt werden, den Ursprung des maladaptiven Reaktionsmusters und die Konsequenzen

61-80 Gute Identifizierung maladaptiver Muster: Der Therapeut markiert deutlich maladaptive Muster, benennt Abwehrmechanismen und die Ängste, die ein bestimmtes Gefühl abwehren. Er exploriert den Ursprung der maladaptiven Reaktionsmuster und/oder deren Auswirkungen, aber es fehlen die kooperative Zusammenarbeit und die abschließende Rekapitulation

41-60 Mäßige Identifizierung der maladaptiven Reaktionsmuster: Der Therapeut beschreibt, dass maladaptive Muster auftreten, benennt einzelne Abwehrmechanismen und Ängste, geht aber nicht auf Ursache und Wirkung ein, und es fehlt die kooperative Zusammenarbeit bei der Umstrukturierung der Abwehr

21-40 Minimales Bewusstsein für die maladaptiven Muster: Der Therapeut weist auf Aspekte maladaptiver Reaktionsmuster hin, benennt einzelne Abwehrmechanismen und/oder Ängste, exploriert aber nicht die Ursachen des Verhaltens, und er bittet den Patienten nicht um Mitarbeit bei der Exploration. Der Patient weist auf problematische Muster hin; der Therapeut validiert die Beobachtungen

Fehlende Identifizierung maladaptiver Muster: Maladaptive Reaktionsmuster werden nicht erfasst oder nicht fokussiert, selbst wenn der Patient sie aufzeigt. Der Therapeut spricht Ängste und Abwehrmechanismen an, jedoch fehlen Fokus und Klarheit. Der Therapeut scheint kein Bewusstsein für die maladaptiven Reaktionsmuster zu haben oder sich gar dagegen zu wehren, selbst wenn der Patient diese verdeutlicht

aHier wird beurteilt, wie gut es dem Therapeut gelingt, die maladaptiven Reaktionsmuster des Patienten (Gefühle, Gedanken, Verhaltensweisen) zu erkennen und darüber in ein therapeutisches Gespräch mit dem Patienten kommen

Mobilisierung der abgewehrten Affekte, das Ausmaß der Angst, der Grad adaptiven Verhaltens (beispielsweise Selbstöffnung, Erleichterung) sowie die Qualität der Selbst- und Objektvorstellung bzw. -wahrnehmung (beispielsweise Spaltung und Mitgefühl). Mehrere empirische Arbeiten haben Reliabilität, Validität und Nützlichkeit der ATOS gezeigt (Berggraf et al. 2014a, 2012; McCullough et al. 2011; Ryum et al. 2014b; Town et al. 2012; Valen et al. 2011). Beispielsweise konnte mithilfe der ATOS abgebildet werden, wie Einsicht positiv mit dem Behandlungsergebnis korrelierte (Kallestad et al. 2010). Eine andere Prozessstudie mit der ATOS zeigte, dass das Ausmaß des emotionalen Erlebens mit der Umstrukturierung der Selbst- und Objektwahrnehmung korrelierte. Je tiefer das emotionale Erleben war, desto mitfühlender und realistischer wurde die Selbst- und Objektwahrnehmung (Berggraf et al. 2014b). Analog zur ATOS entwarf Osborn eine weitere Skala, um die Interventionen des Therapeuten im Sinne einer Adhärenzskala zu beurteilen (Donovan et al. 2016; - Tab. 1): Beurteilt wird auf einer Skala von 1 bis 100, wie gut der Therapeut das maladaptive Verhalten (Abwehr) erkennt und dem Patienten bewusst macht, wie gut er die Motivation des Patienten fördert, das maladaptive Verhalten einzustellen, in welchem Ausmaß er das Erleben der Affekte fördert, Einsicht vermittelt, Angst reguliert sowie die Selbst- und Objektwahrnehmung umstrukturiert (• Tab. 1).

\section{Evidenz}

Die APT konnte wiederholt zeigen, dass Patienten erfolgreich mit diesem Behandlungsmodell behandelt werden können. Es gibt bisher 7 randomisierte kontrollierte Studien (RCT), darunter 5 RCT, die ein internetbasiertes Selbsthilfeprogramm mit minimaler Psychotherapeutenunterstützung evaluierten, und 2 randomisierte klinische Studien zur APT als Einzelpsychotherapie im Gegenübersitzen bei Patienten mit Persönlichkeitsstörungen.

Ins RCT von Winston et al. (1994) zur Psychotherapie von Persönlichkeitsstörungen wurden insgesamt 93 Patienten eingeschlossen. Die damals neu entwickelte APT $(n=31)$ wurde mit einer anderen psychodynamischen Behandlungsgruppe $(n=31)$ und einer Wartegruppe $(n=31)$ verglichen. Die aktiven Gruppen umfassten jeweils 40 Sitzungen. Beide aktiven Bedingungen waren gleich wirksam (Cohen's d 0,96 für die allgemeine Belastungsschwere, gemessen mit dem Global Severity Index [GSI] der Symptom-Check-Liste [SCL90R; Derogatis 1986] im Vergleich vom Beginn zum Ende der Behandlung; McCullough Magill 2009). In der APTGruppe gab es 6 Drop-outs. In einem späteren RCT von Svartberg et al. (2004) wurden 50 Patienten mit Cluster-CPersönlichkeitsstörungen 1:1 auf 2 aktive Behandlungsgruppen randomisiert (Svartberg et al. 2004). Die eine Hälfte der Patienten erhielt eine APT, die andere Hälfte eine kognitive Verhaltenstherapie (CBT), jeweils im Umfang von 40 Sitzungen. Beim Zweijahres-Followup erreichten in der APT-Gruppe 54\% der Patienten eine Remission vs. $42 \%$ in der Kontrollbedingung. Die Unterschiede zwischen APT und CBT waren nicht signifikant. In der APT-Gruppe gab es keinen Drop-out und keinen Patienten, dessen Zustand sich hinsichtlich der allgemeinen Belastungsschwere (GSI der SCL-90R; Derogatis 1986) während der Behandlung verschlechtert hatte. Beide Studien zeigten also insgesamt eine eher niedrige Therapieabbruchrate (insgesamt $11 \%$ ), bei aufgrund von Metaanalysen zu erwartenden 19-60\% (Gries et al. 2020).

Bisher wurden 5 RCT veröffentlicht, die das internetbasierte Selbstmanagementprogramm, das auf der Grundlage der APT entwickelt wurde (Johansson et al. 2012), evaluierten. Im ersten RCT aus Schweden wurden insgesamt 100 Patienten mit depressiven Störungen und Angststörungen 1:1 auf die internetbasierte APT (iAPT) und eine aktive Kontrollbehandlung randomisiert (Johansson et al. 2013). Die Behandlungen dauerten 10 Wochen mit im Mittel 9,5 min Therapeutenkontakt/Woche. Die 
Tab. 2 Studien zur internetbasierten Affektphobietherapie (iAPT)

\begin{tabular}{|c|c|c|c|}
\hline Studie & Patienten & Intervention & Ergebnis \\
\hline $\begin{array}{l}\text { Johansson } \\
\text { et al. (2013) }\end{array}$ & $\begin{array}{l}\text { Patienten mit Depression oder } \\
\text { Angststörungen, die über das } \\
\text { Internet rekrutiert wurden } \\
\text { Land: Schweden }\end{array}$ & $\begin{array}{l}n=50 \text { iAPT } \\
n=50 \text { aktive Kon- } \\
\text { trollbedingung, } \\
\text { supportiver The- } \\
\text { rapeutenkontakt } \\
\text { und Symptom- } \\
\text { Monitoring }\end{array}$ & $\begin{array}{l}\text { Intention-to-treat-Analyse für den Vergleich zur Kontrollbedingung bei Behand- } \\
\text { lungsbeginn zu Behandlungsende: Depression } d=0,77 ; \text { Angst } d=0,48 \\
\text { Outcome-Instrumente: Fragebogen, Fremd-Rating } \\
\text { Adhärenz der iAPT: Drop-out } 0 \text { von } 50 ; 84 \% \text { der Teilnehmer bearbeiteten alle } \\
\text { Module }\end{array}$ \\
\hline $\begin{array}{l}\text { Johansson } \\
\text { et al. (2017) }\end{array}$ & $\begin{array}{l}\text { Soziale Angststörung, Patien- } \\
\text { ten wurden über das Internet/ } \\
\text { Medien rekrutiert } \\
\text { Land: Schweden }\end{array}$ & $\begin{array}{l}n=36 \text { iAPT } \\
n=26 \text { Wartekon- } \\
\text { trollgruppe }\end{array}$ & $\begin{array}{l}\text { Intention-to-treat-Analyse für den Vergleich zur Kontrollbedingung bei Behand- } \\
\text { lungsbeginn zu Behandlungsende: soziale Angst d=1,05; Response 58,3\% vs. } \\
27,8 \% \text {; Remission 27,8\% vs. 11,1\% } \\
\text { Outcome-Instrumente: Fragebogen } \\
\text { Adhärenz iAPT: Drop-out } 1 \text { von 36; im Mittel wurden } 80 \% \text { der Module bearbei- } \\
\text { tet }\end{array}$ \\
\hline $\begin{array}{l}\text { Zwerenz } \\
\text { et al. (2017) }\end{array}$ & $\begin{array}{l}\text { Stationäre psychosomatische Pa- } \\
\text { tienten, Nachsorgebehandlung } \\
\text { (Hauptdiagnosen: Depression, } \\
\text { Angststörungen, Persönlichkeits- } \\
\text { störungen, Depersonalisations- } \\
\text { und Derealisationssyndrom) } \\
\text { Land: Deutschland }\end{array}$ & $\begin{array}{l}n=41 \text { iAPT } \\
n=41 \text { Wartekon- } \\
\text { trollgruppe }\end{array}$ & $\begin{array}{l}\text { Intention-to-treat-Analyse für den Vergleich zur Kontrollgruppe bei Behand- } \\
\text { lungsbeginn zu Behandlungsende: Depression } d=0,60 \text {; Lebensqualität } d=0,53 \\
\text { und emotionale Kompetenz } d=0,49 \text {. } \\
\text { Diejenigen Patienten }(n=13) \text {, die das Selbsthilfeprogramm komplett bearbeitet } \\
\text { hatten, wiesen deutlich stärkere Effekte auf: Depression } d=1,33 \text {, Lebensqualität } \\
d=0,83 \text {, emotionale Kompetenz } d=0,68 \\
\text { Outcome-Instrumente: Fragebogen } \\
\text { Adhärenz der iAPT: Drop-out } 4 \text { von } 41 \text {; nur } 13 \text { von } 41 \text { Patienten ( } 32 \%) \text { hatten } \\
\text { mehr als } 50 \% \text { der Module bearbeitet }\end{array}$ \\
\hline $\begin{array}{l}\text { Lindqvist } \\
\text { et al. }(2020)\end{array}$ & $\begin{array}{l}\text { Adoleszente (Durchschnittsalter } \\
16,6 \text { Jahre) mit der Diagnose } \\
\text { einer Depression, die über das } \\
\text { Internet rekrutiert wurden } \\
\text { Land: Schweden }\end{array}$ & $\begin{array}{l}n=34 \text { iAPT } \\
n=31 \text { aktive Kon- } \\
\text { trollbedingung } \\
\text { (supportiver Thera- } \\
\text { peutenkontakt und } \\
\text { Symptommonito- } \\
\text { ring) }\end{array}$ & $\begin{array}{l}\text { Intention-to-treat-Analyse für den Vergleich zur Kontrollbedingung bei Be- } \\
\text { handlungsbeginn zu Behandlungsende: Depression } d=0,82 \text {, Angst } d=0,78 \text {, } \\
\text { Emotionsregulation } d=0,97 \text {, Selbstmitgefühl } d=0,65 \text {; Response } 56 \% \text { vs. } 21 \% \text {, } \\
\text { Remission } 35 \% \text { vs. } 8 \% \\
\text { Outcome-Instrumente: Fragebogen } \\
\text { Adhärenz der iAPT: Drop-out } 4 \text { von 34, im Mittel wurden 5,8 Module von insge- } \\
\text { samt } 8 \text { bearbeitet ( } 72 \%)\end{array}$ \\
\hline $\begin{array}{l}\text { Lindegaard } \\
\text { et al. (2020) }\end{array}$ & $\begin{array}{l}\text { Soziale Angststörung, Patien- } \\
\text { ten wurden über das Internet/ } \\
\text { Medien rekrutiert } \\
\text { Land: Schweden }\end{array}$ & $\begin{array}{l}n=23 \text { iAPT } \\
n=13 \text { iCBT }\end{array}$ & $\begin{array}{l}\text { Intention-to-treat-Analyse für den Vergleich Behandlungsbeginn zu Behand- } \\
\text { lungsende: soziale Angst } d=0,40 \text {; kein Unterschied zwischen iAPT und iCBT } \\
\text { Outcome-Instrumente: Fragebogen, Fremd-Rating } \\
\text { Adhärenz der iAPT: Drop-out } 2 \text { von } 23 \text {, im Mittel wurden } 80 \% \text { der Module bear- } \\
\text { beitet }\end{array}$ \\
\hline \multicolumn{4}{|c|}{$\begin{array}{l}\text { Die iAPT hatte in den Studien eine Behandlungsdauer von } 8 \text { von } 10 \text { Wochen. Neben den internetbasierten Selbsthilfematerialien gab es eine minimale Thera- } \\
\text { peutenunterstützung }\end{array}$} \\
\hline
\end{tabular}

Kontrollgruppe umfasste das SymptomMonitoring und den supportiven Therapeutenkontakt in gleicher Dosis. Im Vergleich zur aktiven Kontrollgruppe ergaben sich ein starker Effekt für Depressivität $(d=0,77)$ und ein moderater Effekt für Angst $(\mathrm{d}=0,48)$. In der Interventionsgruppe kam es bei $52 \%$ zu einer Remission vs. $24 \%$ in der Kontrollgruppe. Die Effekte waren über eine 7-monatige Nachbeobachtung stabil (Johansson et al. 2013). Weitere 2 RCT untersuchten die Wirkung der iAPT bei sozialen Ängsten (Johansson et al. 2017; Lindegaard et al. 2020), ein RCT bei depressiven Adoleszenten (Lindqvist et al. 2020) und ein RCT die internetbasierte APT als Nachsorgeprogramm für Patienten nach einer stationären psychosomatisch- psychotherapeutischen Krankenhausbehandlung (Zwerenz et al. 2017; • Tab. 2).

Insgesamt stellten alle Studien fest, dass die iAPT von über $80 \%$ der Patienten als gut bis sehr gut bewertet wurde (Johansson et al. 2013, 2017; Lindegaard et al. 2020; Lindqvist et al. 2020; Zwerenz et al.2017). In einer Studie wurde auch die Patientenpräferenz erfasst. Es zeigte sich, dass die meisten Patienten die iAPT der iCBT vorzogen $(63,9 \%$ vs. $36,1 \%$; Lindegaard et al. 2020). In der Nachsorgestudie von Zwerenz et al. (2017) wurde deutlich, dass die Wirksamkeit des Selbsthilfeprogramms stark von Motivation und Adhärenz der Teilnehmer abhängig war (Zwerenz et al. 2017), jedoch auch hier die meisten Patienten die iAPT positiv oder sehr positiv ( $88 \%$ ) bewerteten.
Insgesamt kann festgehalten werden, dass es einige RCT-Ergebnisse gibt, die auf eine Wirksamkeit der APT bei unterschiedlichen Patientengruppen und in unterschiedlichen Settings hinweisen, jedoch umfassen alle genannten RCT eher kleine Stichproben. Bezüglich der Patientenpräferenz existiert bisher nur eine Studie, in der iAPT mit iCBT verglichen wurde, sodass dies keine Verallgemeinerungen hinsichtlich der Patientenpräferenz erlaubt. Weitere Studien mit ausreichender "power" sind notwendig.

\section{Diskussion}

Die APT ist ein evidenzbasierter psychodynamischer Therapieansatz, der das emotionale Erleben in den Mittelpunkt des therapeutischen Prozesses stellt. 
Auch wenn es allgemein anerkannt ist, dass die Arbeit an den „Affekten im Zentrum der tiefenpsychologisch fundierten Psychotherapie“ steht (Rudolf et al. 2018), gibt es doch oft Unsicherheiten, wie konkret mit Emotionen in der Therapie gearbeitet werden kann. Häufig wird über Gefühle mehr gesprochen, als dass die Affekte auch tatsächlich erlebt werden (Kuhn 2014). Dabei ist der Fokus auf das emotionale Erleben von herausragender Bedeutung für den Behandlungsprozess, wie mehrere Prozess-Outcome-Studien zeigen konnten (Friederich et al. 2017; Subic-Wrana et al. 2016; Town et al. 2019, 2017). Die APT bietet hier konkrete Interventionen für die Affektfokussierung an.

Der Fokus auf das emotionale Erleben bildet außerdem eine Schnittstelle mit unterschiedlichen Therapieansätzen. Beispielsweise stellt die APT/iAPT einen wichtigen Referenzpunkt des evidenzbasierten „Unified Protocol“ der psychodynamischen Psychotherapie emotionaler Störungen dar (Leichsenring und Salzer 2014; Leichsenring und Steinert 2018), insbesondere hinsichtlich der Anleitung supportiver Interventionen, der Fokusfindung, Affektfokussierung und der Bearbeitung der Abwehr (Leichsenring und Steinert 2018). Während Mentalisieren pragmatisch als „sich Gedanken und Gefühle vergegenwärtigen“ definiert wird (Schultz-Venrath und Döring 2013), bietet die APT einen Werkzeugkasten mentalisierungsfördernder Interventionen. Die iAPT kann als Psychoedukation betrachtet werden, die v. a. das selbst- und affektbezogene Mentalisieren fördert, indem das Programm hilft, Zusammenhänge zwischen emotionalen Problemen und Symptomen $\mathrm{zu}$ erkennen. Mit kognitiv-behavioralen Ansätzen finden sich die engsten Berührungspunkte mit dem verhaltenstherapeutischen „Unified Protocol zur Behandlung emotionaler Störungen“ (Barlow et al. 2016; Boettcher et al. 2018), das ebenfalls die Vermeidung angstbesetzter Emotionen als zentralen transdiagnostischen Krankheitsmechanismus betrachtet. Der Affektfokus stellt auch eine Brücke zu allgemeinpsychologischen Konzepten dar. So konnte gezeigt werden, dass das psychische
Funktionsniveau bzw. das Niveau der psychischen Integration, wie es in der Operationalisierten Psychodynamischen Diagnostik (Diagnostik AZOP 2006) abgebildet wird, nahezu identisch mit dem Konstrukt der emotionalen Intelligenz ist (Jauk und Ehrenthal 2020). Dies ist ein bemerkenswerter Befund, denn er zeigt erstens die Vereinbarkeit psychodynamischer mit allgemeinpsychologischen Konzepten, und zweitens impliziert er, dass die Besserung des psychischen Funktionsniveaus eine Verbesserung der emotionalen Kompetenzen erfordert. Im Bereich stationärer Psychotherapie kann das APT-Modell hilfreich sein, weil es für schulenübergreifende Teams eine gemeinsame Verständnisbasis bietet. Eine weitere interessante Möglichkeit der APT stellt die Verfügbarkeit eines darauf aufbauenden Selbsthilfeprogramms dar, wodurch „Blended-Care“-Modelle und die internetbasierte Nachsorge erleichtert werden (Zwerenz et al. 2017). Hinsichtlich der Aus- und Fortbildung kann die APT hilfreich sein, weil die Manualisierung praxisnahe und konkrete Anleitungen für Interventionen bietet (McCullough et al. 2019).

Eine Limitation der APT ist, dass bisher nur zwei RCT zu Persönlichkeitsstörungen mit erheblicher Komorbidität vorliegen, und die weiteren klinischen Studien die APT als internetbasierte Selbsthilfe untersuchten. Es gibt also noch Forschungsbedarf. Seitens eines Gutachters wurde die Frage aufgeworfen, ob spezifische Nebenwirkungen mit der APT verbunden sind. Hierzu gibt es gemäß dem Wissen der Autoren keine Forschung. Die bisherigen Studien zeigen aber eine gute Verträglichkeit und Akzeptanz bei den Patienten. Nichtsdestotrotz reagieren nichtwenige Kollegen, wenn sie erstmals das Video einer APTSitzung sehen, mit Sorge, sobald sie Zeuge der ungewohnt aktiven Bearbeitung der Abwehr und raschen Affektmobilisierung werden. Hinsichtlich der Verträglichkeit und Patientenakzeptanz ist es jedoch wichtig zu verstehen, dass in der APT die Affektexposition immer mit der Angstregulation Hand in Hand geht und Patienten sich dadurch sehr gesehen und gehalten fühlen. Limitationen im Kontext einer vertieften Ausbildung in der Methode sind derzeit v.a. die Sprachbarriere. Ausbildungsplattform und zertifizierte Supervision sind ans Englische gebunden. Das Manual und die verfügbaren Lehrvideos vermitteln aber bereits einen lebendigen Eindruck von der Methode, sodass APT-Techniken schrittweise in den eigenen Stil integriert werden können.

\section{Fazit für die Praxis}

\section{- Affekte sind das primäre Motivati- onssystem des Menschen und spielen deshalb für die Genese und Behand- lung psychischer Störungen eine herausragende Rolle. \\ - Die Affektphobietherapie (APT) ist ein psychodynamisches Therapie- modell, das zentrale Elemente der Psychoanalyse (Übertragung, Ab- wehr, Widerstand und Affekte) sowie Verhaltenstherapie (Vermeidung, Exposition und Desensibilisierung) integriert. \\ - Die APT kann aufgrund der prozess- orientierten Manualisierung sowie der Fokussierung auf Interventionen und therapeutische Fertigkeiten das Lernen von Psychotherapie erleich- tern.}

\section{Korrespondenzadresse}

\section{Matthias Michal}

Klinik und Poliklinik für Psychosomatische Medizin und Psychotherapie, Universitätsmedizin Mainz

Langenbeckstr. 1, 55131 Mainz, Deutschland michal@uni-mainz.de

Funding. Open Access funding enabled and organized by Projekt DEAL.

\section{Einhaltung ethischer Richtlinien}

Interessenkonflikt. M. Michal gab an, dass kein Interessenkonflikt besteht. K. Osborn ist zertifizierte Ausbilderin und Supervisorin für die APT.

Für diesen Beitrag wurden von den Autoren keine Studien an Menschen oder Tieren durchgeführt. Für die aufgeführten Studien gelten die jeweils dort angegebenen ethischen Richtlinien.

Open Access. Dieser Artikel wird unter der Creative Commons Namensnennung 4.0 International Lizenz veröffentlicht, welche die Nutzung, Vervielfältigung, Bearbeitung, Verbreitung und Wiedergabe in jegli- 
chem Medium und Format erlaubt, sofern Sie den/die ursprünglichen Autor(en) und die Quelle ordnungsgemäß nennen, einen Link zur Creative Commons Lizenz beifügen und angeben, ob Änderungen vorgenommen wurden.

Die in diesem Artikel enthaltenen Bilder und sonstiges Drittmaterial unterliegen ebenfalls der genannten Creative Commons Lizenz, sofern sich aus der Abbildungslegende nichts anderes ergibt. Sofern das betreffende Material nicht unter der genannten Creative Commons Lizenz steht und die betreffende Handlung nicht nach gesetzlichen Vorschriften erlaubt ist, ist für die oben aufgeführten Weiterverwendungen des Materials die Einwilligung des jeweiligen Rechteinhabers einzuholen.

Weitere Details zur Lizenz entnehmen Sie bitte der Lizenzinformation auf http://creativecommons.org/ licenses/by/4.0/deed.de.

\section{Literatur}

Abbass AA, Kisely SR, Town JM et al (2014) Short-term psychodynamic psychotherapies for common mental disorders. Cochrane Database Syst Rev. https://doi.org/10.1002/14651858.CD004687. pub4

American Psychiatric Association (2013) Diagnostic and Statistical Manual of Mental Disorders: DSM5. American Psychiatric Pub Incorporated

Balint M, Balint E, Ornstein PH (2013) Focal psychotherapy: an example of applied psychoanalysis. Taylor \& Francis, London

Barlow DH, Allen LB, Choate ML (2016) Toward a unified treatment for emotional disorders-republished article. Behav Ther 47:838-853

Berggraf L, Ulvenes PG, Wampold BE et al (2012) Properties of the achievement of therapeutic objectives scale (ATOS): a generalizability theory study. Psychother Res 22:327-347

Berggraf L, Ulvenes PG, Hoffart A et al (2014a) Growth in sense of self and sense of others predicts reduction in interpersonal problems in shortterm dynamic but not in cognitive therapy. Psychother Res 24:456-469

Berggraf L, Ulvenes PG, Oktedalen T et al (2014b) Experience of affects predicting sense of self and others in short-term dynamic and cognitive therapy. Psychotherapy 51:246-257

Boettcher J, Schaeuffele C, Renneberg B (2018) Das Unified Protocol zur Behandlung emotionaler Störungen. Psychotherapeut 63:182-187

Bowlby EJM (2010) Separation: anxiety and anger: attachment and loss Bd. 2. Random House, New York

Cautela JR (1966) Treatment of compulsive behavior by covert sensitization. Psychol Rec 16:33-41

Cautela JR (1973) Covert processes and behavior modification. J Nerv Ment Dis 157:27-36

Davanloo H (2001) Intensive short-term dynamic psychotherapy: selected papers of Habib Davanloo. Wiley, Hoboken, New Jersey

Demos EV (2019) The affect theory of Silvan Tomkins for psychoanalysis and psychotherapy: recasting the essentials. Taylor \& Francis, London

Derogatis LR (1986) SCL 90 R administration, scoring and procedures manual II for the revised version and other instruments of the psychopathology rating scale series. Clinical Psychometric Research, Towson
Diagnostik AZOP (2006) Operationalisierte psychodynamische Diagnostik OPD-2: das Manual für Diagnostik und Therapieplanung. Huber, Mannheim

Donovan JM, Osborn KR, Rice S (2016) Paraverbal communication in psychotherapy: beyond the words. Rowman \& Littlefield, Publishers

Eppel A (2018) Short-term psychodynamic psychotherapy.Springer, Berlin Heidelberg

Fonagy P, Gergely G, Jurist E et al (2010) Affect regulation, mentalization, and the development of the self. Other Press, New York

Freud S (1991) Hemmung, Symptom und Angst. In: Gesammelte Werke: XIV: Werke Aus Den Jahren 1925-1931, S113-205

Friederich HC, Brockmeyer T, Wild B et al (2017) Emotional expression predicts treatment outcome in focal psychodynamic and cognitive behavioural therapy for anorexia nervosa: findings from the ANTOP study. Psychother Psychosom 86:108-110

Grecucci A, Frederickson J, Job R (2017) Editorial: advances in emotion regulation:from neuroscience to psychotherapy. Front Psychol 8:985

Gries S, Longley M, Kästner D et al (2020) Therapeutenmerkmale und Therapieabbruch. Psychotherapeut 65:425-443

Jauk E, Ehrenthal JC (2020) Self-reported levels of personality functioning from the operationalized psychodynamic diagnosis (OPD) system and emotional intelligence likely assess the same latent construct. J Pers Assess. https://doi.org/ 10.1080/00223891.2020.1775089

Johansson R, Hesser H, Ljotsson B et al (2012) Transdiagnostic, affect-focused, psychodynamic, guided self-help for depression and anxiety through the internet: study protocol for a randomised controlled trial. BMJ Open. https://doi.org/10. 1136/bmjopen-2012-002167

Johansson R, Bjorklund M, Hornborg C et al (2013) Affect-focused psychodynamic psychotherapy for depression and anxiety through the Internet: a randomized controlled trial. Peer 1 1:e102

Johansson R, Hesslow T, Ljotsson B et al (2017) Internetbased affect-focused psychodynamic therapy for social anxiety disorder: a randomized controlled trial with 2-year follow-up. Psychotherapy 54:351-360

Kallestad H, Valen J, Mccullough L et al (2010) The relationship between insight gained during therapy and long-term outcome in short-term dynamic psychotherapy and cognitive therapy for cluster $\mathrm{C}$ personality disorders. Psychother Res 20:526-534

Kuhn N (2014) Intensive short-term dynamic psychotherapy: a reference. Createspace Independent Pub, North Charleston

Leichsenring F, Salzer S (2014) A unified protocol for the transdiagnostic psychodynamic treatment of anxiety disorders: an evidence-based approach. Psychotherapy 51:224-245

Leichsenring F, Steinert C (2018) Towards an evidencebased unified psychodynamic protocol for emotional disorders.J Affect Disord 232:400-416

Lindegaard T, Hesslow T, Nilsson Metal (2020) Internetbased psychodynamic therapy vs cognitive behavioural therapy for social anxiety disorder: A preference study. Internet Interv 20:100316

Lindqvist K, Mechler J, Carlbring P et al (2020) Affect-focused psychodynamic Internet-based therapy for adolescent depression: randomized controlled trial. J Med Internet Res 22:e18047

Malan DH (2013) A study of brief psychotherapy. Taylor \&Francis, London
McCullough JP (1984) Cognitive-behavioral analysis system of psychotherapy: an interactional treatment approach for dysthymic disorder. Psychiatry 47:234-250

McCullough Vaillant L (1997) Changing character: short term anxiety-regulating psychotherapy for restructuring defense. Basic Books, New York

McCullough L, Magill M (2009) Affect-focused short-term dynamic therapy. In: Levy RA, Ablon JS (Hrsg) Handbook of evidence-based psychodynamic psychotherapy: bridging the gap between science and practice. Humana Press, Totowa, S249-277

McCullough L, Osborn KA (2004) Short term dynamic psychotherapy goes to Hollywood: the treatment of performance anxiety in cinema. J Clin Psychol 60:841-852

McCullough L, Kuhn N, Andrews S (2003) Treating affect phobia: A manual for short-term dynamic psychotherapy. Guilford, New York

McCullough L, Bhatia M, Ulvenes P et al (2011) Learning how to rate video-recorded therapy sessions: a practical guide for trainees and advanced clinicians. Psychotherapy 48:127-137

McCullough L, Kuhn N, Andrews S et al (2019) Affektfokussierte psychodynamische Psychotherapie: Ein integratives Manual zur Behandlung von Affektphobien. Kohlhammer, Stuttgart

Rudolf G, Wöller W, Kruse J (2018) Tiefenpsychologisch fundierte Psychotherapie: Basisbuch und Praxisleitfaden. Schattauer, Stuttgart

Ryum T, Store-Valen J, Svartberg Met al (2014a) Factor analysis of the Achievement of Therapeutic Objectives Scale (ATOS) in short-term dynamic psychotherapy and cognitive therapy. Psychol Assess 26:925-934

Ryum T, Støre-Valen J, Svartberg M et al (2014b) Factor analysis of the achievement of therapeutic objectives scale (ATOS) in short-term dynamic psychotherapy and cognitive therapy. Psychol Assess 26:925-934

Schultz-Venrath U, Döring P (2013) Lehrbuch Mentalisieren: Psychotherapien wirksam gestalten. Klett-Cotta, Stuttgart

Subic-Wrana C, Greenberg LS, Lane RD et al (2016) Affective change in psychodynamic psychotherapy: theoretical models and clinical approaches to changing emotions. Z Psychosom Med Psychother62:207-223

Svartberg M, Stiles TC, Seltzer MH (2004) Randomized, controlled trial of the effectiveness of shortterm dynamic psychotherapy and cognitive therapy for cluster C personality disorders. Am J Psychiatry 161:810-817

Tomkins SS, Demos EV, Smith B (1995) Exploring affect: the selected writings of Silvan S tomkins. Cambridge University Press, Cambridge

Town JM, Hardy GE, Mccullough L et al (2012) Patient affect experiencing following therapist interventions in short-term dynamic psychotherapy. Psychother Res 22:208-219

Town JM, Salvadori A, Falkenström F et al (2017) Is affect experiencing therapeutic in major depressive disorder? Examining associations between affect experiencing and changes to the alliance and outcome in intensive short-term dynamic psychotherapy. Psychotherapy 54:148-158

Town JM, Lomax V, Abbass AA et al (2019) The role of emotion in psychotherapeutic change for medically unexplained symptoms. Psychother Res 29:86-98

Vaillant GE (2011) Involuntary coping mechanisms: a psychodynamic perspective. Dialogues Clin Neurosci 13:366-370 
Vaillant LM (1997) Changing character: shortterm anxiety-regulating psychotherapy for restructuring defenses, affects, and attachment. Basic Books, New York

Valen J, Ryum T, Svartberg M et al (2011) The Achievement of Therapeutic Objectives Scale: interrater reliability and sensitivity to change in short-term dynamic psychotherapy and cognitive therapy. Psychol Assess 23:848-855

Wachtel PL (1977) Psychoanalysis and behavior therapy:toward an integration. Basic Books, New York

Winston A, Laikin M, Pollack J et al (1994) Short-term psychotherapy of personality disorders. Am J Psychiatry 151:190-194

Zwerenz R, Becker J, Johansson R et al (2017) Transdiagnostic, Psychodynamic Web-Based Self-Help Intervention Following Inpatient Psychotherapy: Results of a Feasibility Study and Randomized Controlled Trial. JMIR Ment Health 4:e41.https://doi.org/10.2196/mental.7889.

\section{Auch künstliche neuronale Netze lernen im Schlaf}

Forschende der Technischen Universität Ilmenau haben wegweisende Fortschritte im Bereich des maschinellen Lernens erzielt. In einer Studie wurde gezeigt, wie die Wirkmechanismen, die es Menschen ermöglichen, Erlerntes im Schlaf zu vertiefen, auf künstliche neuronale Netze übertragen werden können.

Erst vor wenigen Jahren konnten Schlafforscher in Experimenten nachweisen, dass der Mensch in der Tiefschlafphase lernt. Die Wissenschaftler bauten auf einer Beobachtung auf, die sie während der Wachphase von Menschen machten: Die Verbindungen zwischen den Synapsen lernen nicht nur aktiv, sondern verstärken oder reduzieren auch chemische oder elektrische Signale der Neuronen, der Nervenzellen. Die Synapsen leiten also Signale nicht nur von Nervenzelle zu Nervenzelle weiter, sondern verstärken auch deren Intensität oder schwächen sie ab. Auf diese Weise versetzen die Synapsen die Neuronen in die Lage, die sich ändernden Einflüsse der Umgebung aufzunehmen und sich ihnen anzupassen.

Im Schlaf normalisiert sich dieser Erregungszustand wieder und das Nervensystem kann die in der aktiven Wachphase aufgenommenen neuen Informationen im Gedächtnis verarbeiten und durch Vergessen zufälliger oder unwichtiger Informationen das Gelernte verfestigen und gleichzeitig für die Aufnahme neuer Informationen empfindlicher werden.

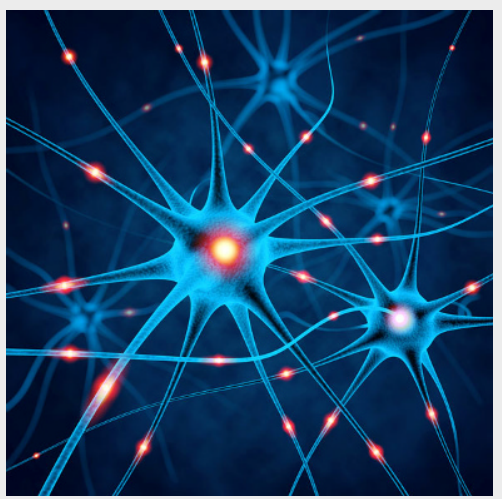

() ag visuell / stock.adobe.com

Auf diesen Vorgang, den Fachleute als synaptische Plastizität bezeichnen, baute Professor Patrick Mäder, Leiter des Fachgebiets Softwaretechnik für sicherheitskritische Systeme der TU IImenau, auf: „Die synaptische Plastizität ist für die Funktion und Leistungsfähigkeit unseres Gehirns verantwortlich und damit die
Grundlage des Lernens.

Würden die Synapsen immer in einem aktivierten Zustand bleiben, würde dies, wie wir aus Tierversuchen wissen, letztlich das Lernen erschweren. Erst die Erholungsphase während des Schlafs macht es möglich, dass wir das, was wir gelernt haben, im Gedächtnis behalten."

Die Fähigkeit des synaptischen Systems, dynamisch auf unterschiedlichste Reize reagieren zu können und das Nervensystem stabil und im Gleichgewicht zu halten, ahmen die Forscher der TU IImenau in künstlichen neuronalen Netzwerken nach. Mithilfe der so genannten synaptischen Skalierung übertragen sie die Mechanismen, die das dynamische System Gehirn regulieren, auf Verfahren des maschinellen Lernens - mit dem Ergebnis, dass sich die künstlichen neuronalen Modelle ähnlich wirkungsvoll verhalten wie ihr natürliches Vorbild.

Anwendungen für solche hocheffizient arbeitenden selbst lernenden Maschinen finden sich etwa in der Medizin, wenn mit Hilfe zum Beispiel von EEG- oder Tomographieuntersuchungen Krankheiten auf der Basis biologischer Daten erkannt werden. Weitere Anwendungsgebiete sind etwa die Smart-grid-Regelung elektrischer Netze oder die automatisierte LaserFertigung.

Publikation: M. Hofmann and P. Mäder, "Synaptic Scaling--An Artificial Neural Network Regularization Inspired by Nature," in IEEE Transactions on Neural Networks and Learning Systems, doi: 10.1109/TNNLS.2021.3050422

Quelle: Technische Universität Ilmenau [www.tu-ilmenau.de, 14.04.2021]) 